\title{
Propagation Characteristics of UWB Radio in a High-Rise Apartment
}

\author{
Simon S.M. Wong \\ Technical Services Division \\ Hong Kong SAR Government, Hong Kong \\ Email: s.m.wong@polyu.edu.hk
}

\author{
Francis C.M. Lau and Chi K. Tse \\ Department of Electronic \& Information Engineering \\ Hong Kong Polytechnic University, Hong Kong \\ Email: \{encmlau, encktse\} @ polyu.edu.hk
}

\begin{abstract}
Among many other applications, ultra wideband (UWB) is considered a promising technology to be deployed for the future wireless personal area networks (WPAN). The objective of our work is to investigate the indoor propagation characteristics of a UWB signal of which its equivalent isotropically radiated power (EIRP) is limited by the Federal Communications Commission (FCC) of the U.S. in [1]. A $90 \mathrm{~m}^{2}$ residential flat in a high-rise building of Hong Kong is a typical example to exhibit the scenarios for a WPAN application. This paper presents the empirical results from various aspects of the propagation characteristics by employing a pair of FCC-compliant UWB transceivers.
\end{abstract}

Keywords - Ultra wideband, channel characterization, path loss model, indoor propagation.

\section{Introduction}

FCC was the pioneer organization who gave definitions and rulings of UWB technology. To avoid interference to the existing radio services, they published the First Report and Order in [1] to limit the UWB radiation and also permitted the technology commercialization. The document defines the radiation masks as shown on Fig. 1

The radio transceivers employed in this experiment operate at the centre frequency of $4.7 \mathrm{GHz}$ with a bandwidth and radiation power of $3.2 \mathrm{GHz}$ and $-11 \mathrm{dBm}$ respectively. They were FCC-compliant UWB devices and were used for various types of analysis throughout the experiment. Its occupied spectrum under the FCC defined mask is also shown on Fig. 1.

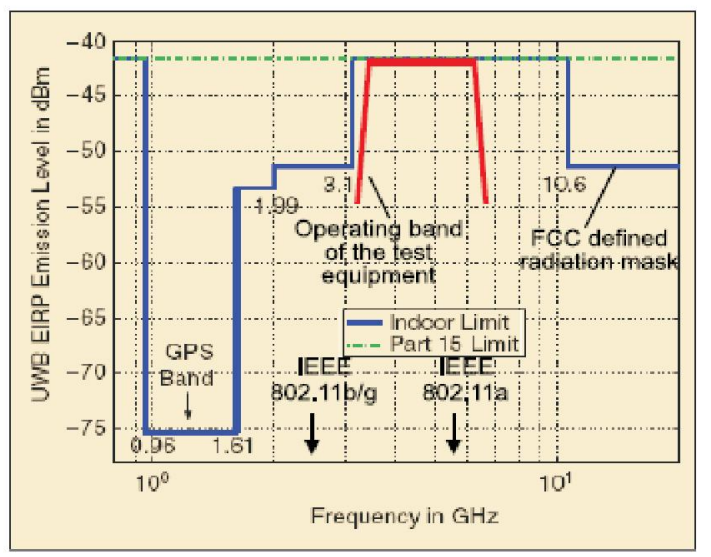

Figure 1. UWB Radiation Mask Defined by the FCC and the Operating Spectrum of the Equipment in Use
UWB technology for WPANs or more specifically wireless body area networks (WBAN), cannot be successfully implemented in the household environment without a thorough analysis of its indoor propagation characteristics. IEEE standardized a channel model in 2003 for indoor UWB communications in their IEEE 802.15.3a Task Group [2]. This channel model was mainly derived for short ranges of less than $10 \mathrm{~m}$. Until recently, another subcommittee under IEEE 802.15.4a Task Group has been formed to study and develop a channel model for longer operating ranges [3].

In addition to the above globally standardized models, a number of literatures have been published to report the field measurements of UWB propagation in indoor environments. Ref. [4] to [8] are the more recent ones. They mostly worked on either low-rise houses or office/industrial premises, but not for the flats or apartments of a high-rise building which are the most popular residential constructions in the highly congested cities like Hong Kong. Ref. [9] is one of the few literatures dedicated for the apartment-type of the indoor environment in Korea. The structural walls in a high-rise building are considered radio unfriendly. The strong and thick partitions made of concrete and steel studs considerably attenuate radio frequencies especially in the higher frequency bands as those used in the UWB. From our experiment, we have derived the path loss exponents $n$ and standard deviations $\sigma$ in different scenarios, i.e. line-of-sight (LOS), non-LOS (NLOS), and corridor. With the waveform scan on the time domain, the time dispersion parameters as a result of multipath can be characterized. We also briefly took a simple signal loss measurement for the radio path obstructed by a door made of glass and wooden frame. It was found that the wall partitions introduce great attenuation to the UWB propagation, and render the UWB signals completely blocked by more than one structural walls.

Human body is also considered radio unfriendly. Ref. [10] and [11] have reported the adverse effects of human body on UWB signals by means of a vector network analyzer. In our investigation, we simulated a body-worn operation environment by belt-mounting a UWB transceiver at the waist level. Not surprisingly, the measured field strengths exhibited a sharp and pronounced null when the orientation of the human body was at an angle that completely blocked the propagation path.

The next section describes in details specifications of the measuring tools, equipment setup, indoor scenarios, and experiment procedures. Section 3 described the measurement 
results and with our analysis and observations from different aspects. Section 4 concludes our findings and suggests some possible areas for further study.

\section{Equipment Setup and Procedures}

\section{A. Test Equipment}

The core equipment employed in our experiment is a UWB Reference Design kit developed by the Time Domain Corporation. The hardware comprises mainly a pair of UWB radio transceivers based on Time Domain's PulsON 210 chipset. Each transceiver is directly mounted with an omni-directional antenna on the front panel of its case. The reference design kit also carries a bundle of software and documents primarily designed for product development. Its separately sold System Analysis Module [12] is a software tool for investigations into the radio performance parameters.

The radiation element is a V-shaped theoretically omni-directional wideband and vertically polarized antenna covering the whole frequency range. However, there is inevitably a $6 \mathrm{~dB}$ variation for the radiation pattern on the $\mathrm{H}$-plane. To minimize this variation affecting the accuracy of our results, the broad sides of respective antennas were always kept facing and parallel to each other such that the antenna gain could be constantly maintained at its maximum throughout our experiment.

\section{B. Environment}

The indoor environment under evaluation is a living flat of approximately $90 \mathrm{~m}^{2}$ in floor area. The flat is on the upper floor of a high-rise residential building that utilizes some of the internal partition walls as structural support. As shown in Fig. 3, the walls in dots are structural walls made of thick concrete materials and with a framework of steel studs inside. Other internal walls are either made of bricks or plasterboards. The flat is fully furnished with all necessary living necessities. The measurements were conducted in mid-night to minimize the effects from any moving objects and electromagnetic interferences caused by the operation of any electrical appliances. All room doors were closed except the glass door at the entrance of the corridor. To resemble the real case of using the UWB devices at waist level, both the transmitter and receiver were placed at $1 \mathrm{~m}$ above the floor. To ensure the environment was free from interference in the interested frequency range, a scan of the ambient noise background was conducted and the result was at an acceptable level as shown on Fig. 2.

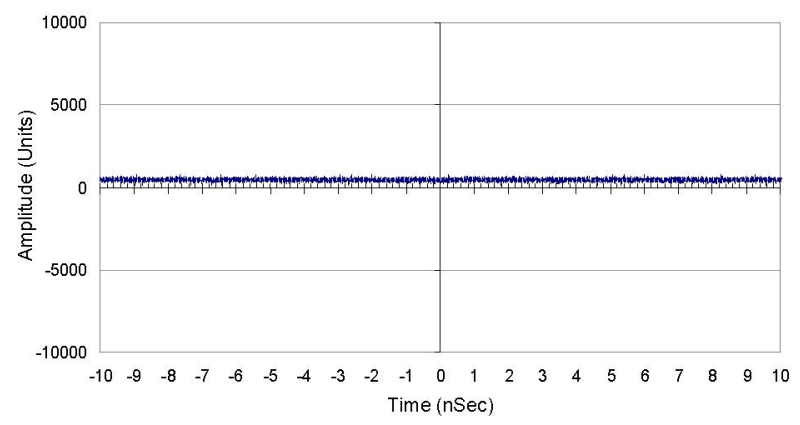

Figure 2. Wavform Scan of Indoor Ambient Noise
Table 1. Different Scenarios of Measurements

\begin{tabular}{|c|c|c|c|c|}
\hline \multirow{2}{*}{ Scenario } & Environment & $\begin{array}{c}\text { Tx } \\
\text { Location }\end{array}$ & $\begin{array}{c}\mathrm{Rx} \\
\text { Location }\end{array}$ & $\begin{array}{c}\text { Tx-Rx } \\
\text { Distance }\end{array}$ \\
\hline 1 & LOS (Living Room) & 1 & $\mathrm{~A}-\mathrm{F}$ & $3.2-6.2 \mathrm{~m}$ \\
2 & NLOS & 1 & $\mathrm{G}-\mathrm{J}$ & $2.6-6.2 \mathrm{~m}$ \\
3 & LOS (Corridor) & 2 & $\mathrm{~K}-\mathrm{N}$ & $3.2-6.6 \mathrm{~m}$ \\
4 & Thro' Glass Door & 2 & $\mathrm{~L}$ & $4.3 \mathrm{~m}$ \\
5 & On Human Body & 1 & $\mathrm{~A}$ & $3.2 \mathrm{~m}$ \\
\hline
\end{tabular}

Table 1 summaries the scenarios in which the measurements were conducted.

\section{Time Dispersion Parameters}

The time dispersion or echoes caused by multipath propagation delays is one of the key areas to be investigated for the understanding of the behaviour of UWB propagation in the flat. The temporal dispersive properties of the multipath channels are commonly characterized by the mean excess delay and root mean square (rms) delay spread as described in [13].

\section{Path Loss Model}

For each scenario, the transmitter was kept stationary and only the receiver was moved to different locations in the flat to collect the measurement data for characterization of the large-scale path loss behaviour of the propagation channel. In order to characterize the small-scale fading effect, at each test point the receiver was moved $25 \mathrm{~mm}$ for 16 locations on a $4 \times 4$ grid, as illustrated by the square grid in Figure 3 . This spacing is approximately equal to $\lambda / 2=24 \mathrm{~mm}$ of the UWB signal with its maximum frequency at $6.3 \mathrm{GHz}$. So, the path loss at each test point can be determined by averaging the corresponding set of spatial path losses by (1).

$$
P L(d)=\frac{\sum_{i=1}^{16} P L_{s p}^{i}(d)}{16}
$$

where $d$ is the distance between transmitter and receiver, $P L_{\mathrm{sp}}^{\mathrm{i}}(d)$ the spatial path-loss at the receiving point with a distance $d$ from the transmitter and at grid point $i$.

By waveform scan the peak amplitude of the received signal at distance $d$ and grid point $i$ and comparing that value to the peak amplitude at the reference position, test point $A$ in this case, the excess path loss (in $\mathrm{dB}$ ) with reference to $1 \mathrm{~m}$ can be expressed in (2) as given by [12].

$$
P L(d)=20 \log \left(\frac{\left|V_{\max }(d)\right|}{\left|V_{\max \operatorname{Rc}\left(d_{A}\right)}\right|}\right)-20 \log \left(\frac{d_{A}}{1}\right), \quad d_{A} \geq 1
$$

where $V_{\max }(d)$ is the peak amplitude of the received waveform at a location with a distance $d$ in metre from the transmitter; the peak amplitude of the received waveform at the reference location, i.e. point $A ; d_{A}$ the distance of the reference point $A$ from the transmitter.

To avoid the receiver being driven into compression that might produce inaccurate results, the waveforms and other parameters were closely monitored when the distance of the receiver was too close to the transmitter. A minimum of $3.2 \mathrm{~m}$ for a clear LOS propagation channel is recommended in [12]. 


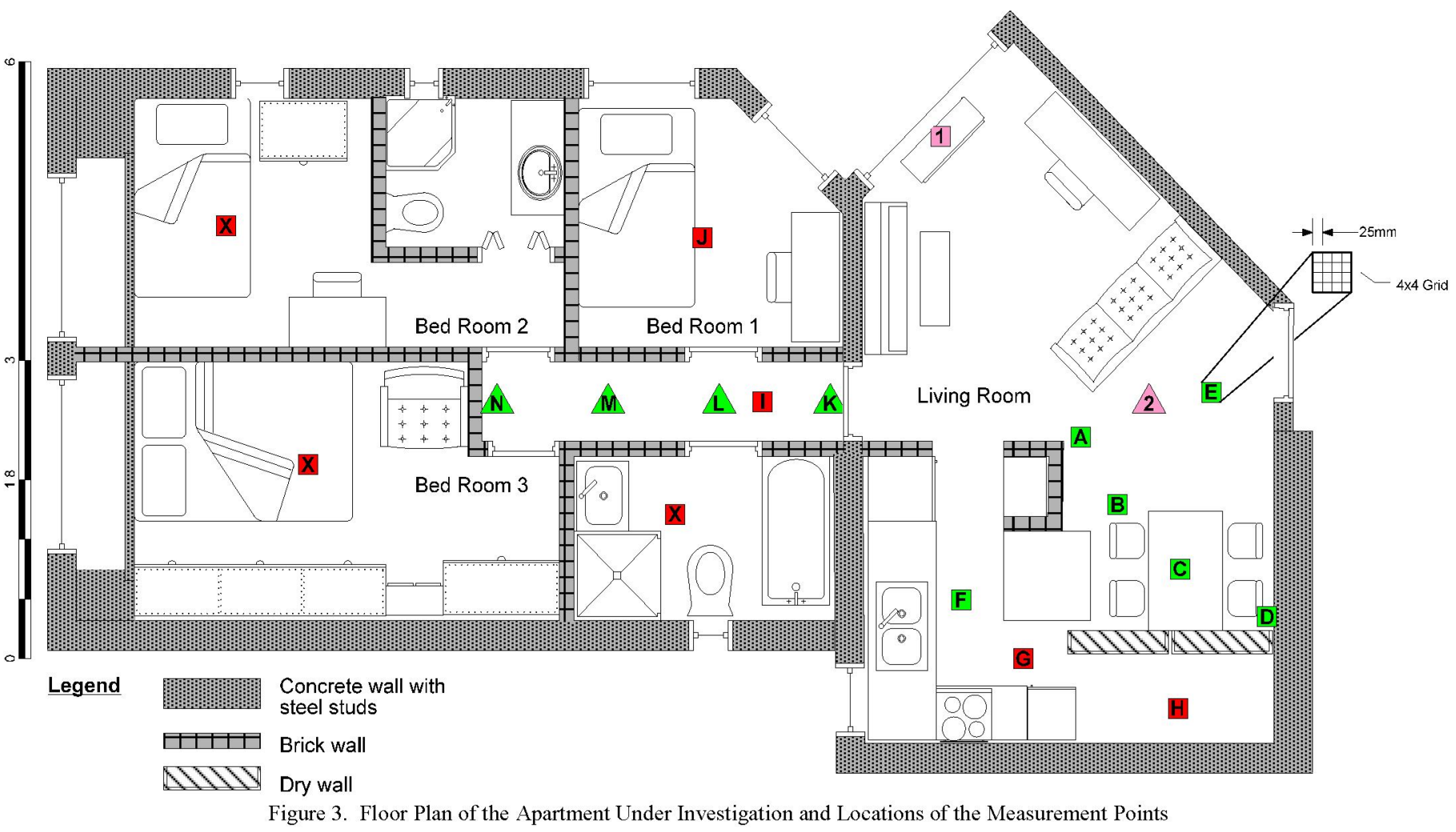

Through actual field measurements, the empirical approach adopted here has the advantage of taking all propagation factors into account. The propagation model is based on the log-distance path loss model as described in [13]. Such model has been extensively used for both narrow and recently wide band transmissions. The model can be realized by curve fitting on a scatter plot of measured data and then derive the path loss exponent $n$ from (3).

$$
\overline{P L}(d)=\overline{P L}\left(d_{0}\right)+10 n \log \left(\frac{d}{d_{0}}\right) \quad d \geq d_{0}
$$

where $\overline{P L}(d)$ is the average large-scale path loss in $\mathrm{dB}$ at a transmitter-receiver distance of $d$ (in metre); $\overline{P L}\left(d_{0}\right)$ is the average large-scale path loss at a reference distance $d_{0}$. When plotted on a log-distance graph, the modeled path loss is a straight line and $n$ can be determined from the slope of $10 n \mathrm{~dB}$ per decade. The small-scale fading effect can also be characterized by the shadowing fading parameter $X_{\sigma}$ and given by (4).

$$
P L(d)=\overline{P L}\left(d_{0}\right)+10 n \log \left(\frac{d}{d_{0}}\right)+X_{\sigma}
$$

where $P L(d)$ is the measured path loss at a particular location; $X_{\sigma}$ is a zero-mean Gaussian distributed random variable (in $\mathrm{dB}$ ) with standard deviation $\sigma$ (in $\mathrm{dB}$ ).

The value of $n$ and $\sigma$ depends on the specific propagation environment. The main objective of our experiment for scenarios 1 to 3 is to determine $n$ and $\sigma$ of a UWB signal propagated in this typical residential environment of a highly congested city.
Scenario 4 was to further investigate the attenuation of a UWB signal through a glass door by simply comparing the measured field strengths with the door open and close. The interaction of human body with UWB signal was measured in scenario 5. The receiver was placed at waist level and in close proximity with an adult human body. A measurement was taken for an angular movement of every rotation of $36^{\circ}$. So, a set of 10 measured data could be obtained and was plotted on a radar chart to show the effect of a human body at different body orientations.

\section{Results and Analysis}

\section{A. Time Dispersion}

A typical waveform of the received LOS UWB signal in the time domain at location $A$ was captured and shown in Fig. 4. Multipath phenomenon can be seen from the temporal dispersive properties. The most dominant multipath component is at 4 nsec delayed after the main LOS component. This excess delay of $4 \mathrm{nsec}$ implies a traveling distance of 1.2 metre which is in agreement with the dimensions of the flat and the furniture inside.

In order to determine an average small-scale power delay profile, Fig. 4 should be represented as plot of relative received power (in $\mathrm{dB}$ ) as a function of excess delay with respect to a fixed time delay reference as shown on Fig. 5. The mean excess delay and rms delay spread for the power delay profile can be computed as follows:

Mean excess delay $\bar{\tau}=5.5 \mathrm{nsec}$;

Rms delay spread $\sigma_{\tau}=2.1 \mathrm{nsec}$ 


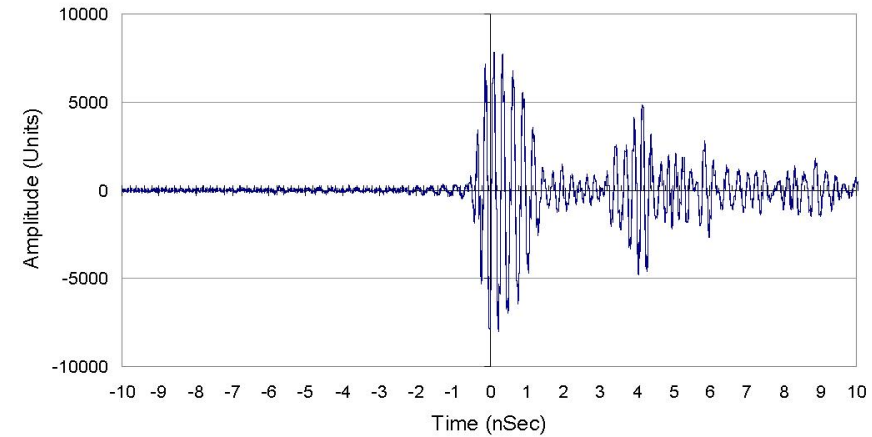

Figure 4. Waveform Captured at Location $A$

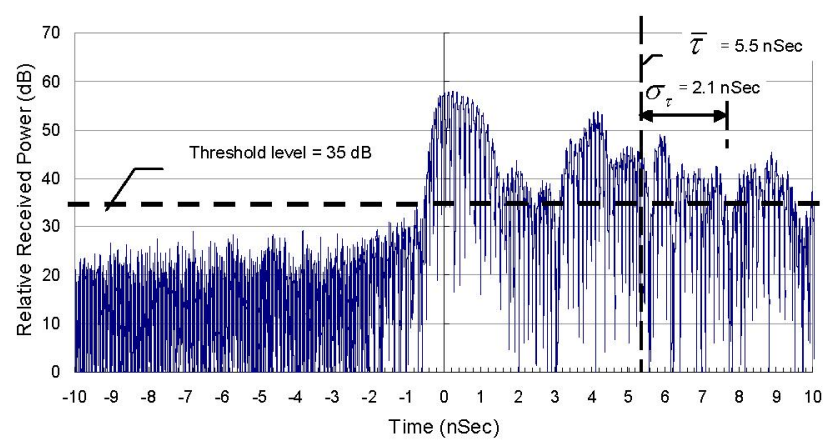

Figure 5. Power Delay Profile of the Signal Received at Location $A$

These values are also indicated on Fig. 5 for a better appreciation of their relative positions on the time domain.

\section{B. Path Loss Model}

The measured path loss results of the 5 scenarios are all plotted on Fig. 6 . Using the linear regression technique described above, the UWB propagation in scenarios 1 and 3 is modeled by the log-distance path loss model and the results are plotted as the corresponding straight lines shown on the
Table 2. Path loss exponents $\boldsymbol{n}$ and standard deviation $\boldsymbol{\sigma}$ for 2 scenarios

\begin{tabular}{|c|c|c|c|}
\hline Scenario & Environment & $n$ & $\sigma$ \\
\hline 1 & LOS (Living Room) & 1.6 & $0.7 \mathrm{~dB}$ \\
3 & LOS (Corridor) & 1.6 & $2.1 \mathrm{~dB}$ \\
\hline
\end{tabular}

same graph. The path loss exponent $n$ and standard deviation $\sigma$ can be computed and shown in Table 2 . It is found that the path loss exponents inside the premises are almost the same ( $n$ $=1.6$ ), no matter the environment is the living room or the corridor. Their standard deviations are 0.7 and $2.1 \mathrm{~dB}$ respectively.

Having compared with other indoor environments covered by [5] - [9] and [14], the floor area of the flat under evaluation is inherently very small. Although the number of test samples is relatively less than theirs, the results of path loss exponents and standard deviations obtained in this experiment agree very much with those published in [6], [9] and [14].

There is an unusual finding that the path loss curve for NLOS propagation exhibits a negative slope as seen on the upper part of Fig. 6. It is because there are extreme attenuation factors due to different building materials at different test points. For example, the nearest test point $J$ gives the highest path loss because of the concrete structural wall it passed through. On the contrary, the farthest test point $H$ exhibits the least loss because the partition in between the transmitter and receiver is made of a soft plasterboard material. So, the path loss exponent of NLOS in this case is misleading and therefore has not been computed.

\section{Partition and Human Body Losses}

There is a wide variety of partitions and obstacles to form the internal and external structures of the apartment. Partitions vary widely in their physical and electrical characteristics and therefore exhibit extreme attenuation performance for radio waves propagation. It is particularly true for the higher

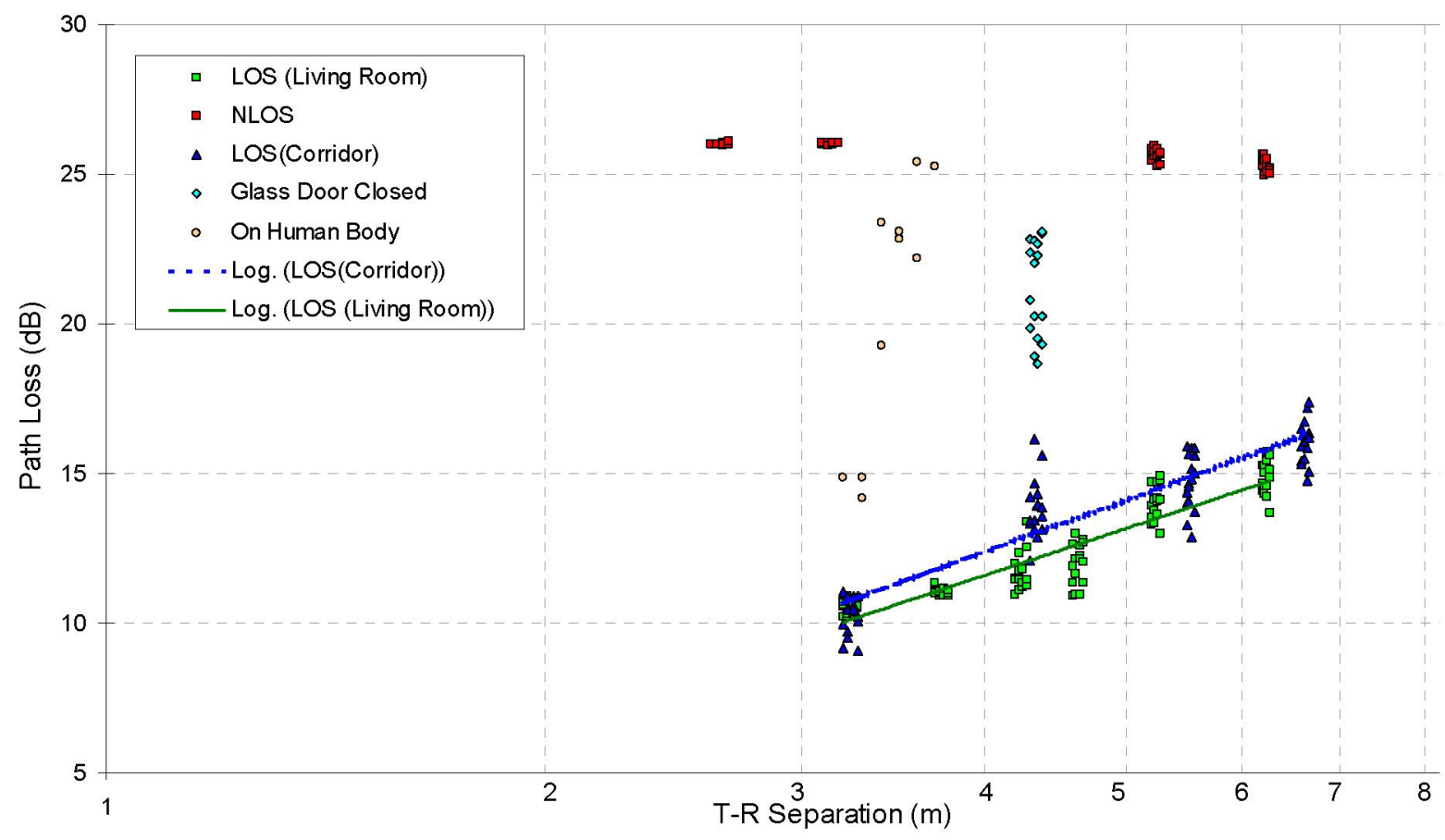

Figure 6. Scatter Plot of Measured Data and Log-Distance Path Loss Model 
Table 3. Propagation Loss Due to the Effect of Different Materials

\begin{tabular}{|c|c|}
\hline Materials & Average Signal Loss \\
\hline Concrete Wall & $19 \mathrm{~dB}$ \\
Dry Wall & $10 \mathrm{~dB}$ \\
Glass Door & $9 \mathrm{~dB}$ \\
Human Body & $11 \mathrm{~dB}$ \\
\hline
\end{tabular}

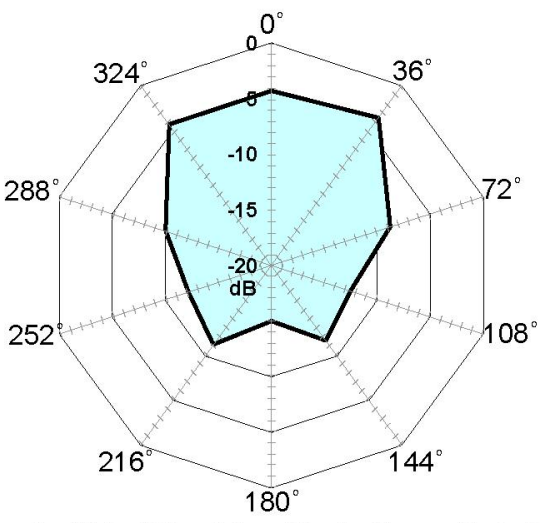

Figure 7. Angular Plot of Signal Loss Due to Human Body With Respect to Clear LOS at the Same Location

frequency narrowband signals as given in [13]. The attenuation performance for UWB, however, has not yet been extensively explored. In this experiment, we have measured the UWB propagation losses of some of the building materials used in the flat as well as its interaction with a human body. The average results are shown in Table 3 and the measurements are also graphically presented on Fig. 6 .

We attempted to take the measurements inside the bedrooms 2, 3 and the washrooms, but no signal was received and the blind spots were indicated by an "X" on Fig. 3. It implies that if a UWB transmitter is placed at location $A$ like a TV set top box for transmitting a video programme, user may not be able to enjoy the programme at these places.

The measurements of propagation loss with human interaction were conducted at the location $A$. The results are plotted angularly on Fig. 7. The data are propagation loss in $\mathrm{dB}$ and with respect to the measured results at the same location with clear LOS. Our measurements on human body also clearly indicate that orientation of the body, and hence the degree of interaction of the human body on the propagation path, affects considerably the received signal. When the path is completely blocked by the human body, i.e. $180^{\circ}$ as shown in Fig. 7, there appears a very sharp and pronounced null.

\section{Conclusion}

We have presented an empirical analysis of the UWB propagation channel in an apartment by using 5 sets of data measured under different scenarios. Our measurement procedures have taken into account both the small-scale and large-scale fading characteristics. The results of both time dispersion measurement and path loss modeling obtained in this experiment for such a typical small flat in Hong Kong are in fact comparable with those for larger premises. We also presented the results of the propagation loss penetrated through different building materials and with the body's effect.
There are approximately $9 \sim 19 \mathrm{~dB}$ excess losses due to these factors.

In order to develop a reliable UWB product for WPAN applications, however, there are still many researches to be done. A good understanding of the UWB propagation channel is essential for the link budgeting. One of the mysterious areas is the frequency dependency of the path loss models. Another area is the comprehensive measurements on signal loss due to different building materials.

\section{REFERENCES}

[1] FCC, First Report and Orders in the Matter of Revision of Part 15 of the Commission's Rules Regarding Ultra Wideband Transmission Systems, ET-Docket 98-153, FCC 02-48, 22 Apr 2002.

[2] J. Foerster, Channel Modeling Sub-committee Report (Final), IEEE p802.15-02/490r1-SG3a, Feb 2003.

[3] A.F. Molisch, et al., IEEE 802.15.4a Channel Model - Final Report, IEEE 802.15-04-0662-00-004a, Nov 2004.

[4] J. Foerster, Q. Li, UWB Channel Modeling Contribution from Intel, IEEE P802.15-02/445r1-SG3a, Jun 2002.

[5] M. Pendergrass, W.C. Beeler, Empirically Based Statistical UWB Channel Model, IEEE P802.15-02/240SG3a, Jul 2002.

[6] S. Ghassemzadeh, V. Tarokh, "UWB Path Loss Characterization in Residential Environments", 2003 IEEE Radio Frequency Integrated Circuits Symposium, 2003

[7] Q. Li, W. Wong, "Measurement and Analysis of the Indoor UWB Channel", 2003 Vehicular Technology Conference, VTC, Fall 2003.

[8] S. Ghassemzadeh, R. Jana, C. Rice, W. Turin, V. Tarokh, "Measurement and Modeling of an Ultra-wide Bandwidth Indoor Channel", IEEE Trans. Communication, Vol. 52, No. 10, pp. 1786-1796, Oct 2004

[9] C. Chong, Y. Kim, S. Lee, "Statistical Characterization of the UWB Propagation Channel in Various Types of High-Rise Apartments", IEEE Communications Society, WCNC 2005.

[10]T. Welch, et al., "The Effects of the Human Body on UWB Signal Propagation in an Indoor Environment", IEEE Journal on Selected Areas in Communications, Vol. 20, No. 9, Dec 2002

[11]S. Promwong, W. Hachitani, G. Ching, J. Takada, "Characterization of Ultra-Wideband Antenna with Human Body", International Symposium on Communications and Information Technologies 2004, ISCIT 2004, Oct 2004.

[12] System Analysis Module User's Manual PulsON 210 UWB Reference Design, Time Domain Corporation, P210-320-0102B, Aug 2005.

[13]T. Rapaport, Wireless Communications: Principles and Practice, Prentice Hall PTR, 2nd Edition, 2002.

[14]S. Ghassemzadeh, R. Jana, C. Rice, W. Turin, Vahid Tarokh, "A Statistical Path Loss Model for In-Home UWB Channels", 2002 IEEE Conference on Ultra Wideband Systems and Technologies, 2002 